\title{
Guidelines and recommendations on the prevention and control of hepatitis C
}

\author{
THE LABORATORY CENTRE FOR DISEASE CONTROL
}

\section{GENERAL INFORMATION}

\subsection{EPIDEMIOLOGY}

In 1993, 1635 cases of hepatitis C virus (HCV) were reported to Health Canada's Laboratory Centre for Disease Control (LCDC). These data include reports from only seven provinces/territories, and both acute and chronic cases were included.

In the LCDC's Sentinel Health Unit Surveillance System 305 newly identified cases of hepatitis C (both acute and chronic) were identified in 1994. Of these individuals 28\% had previous blood transfusion as a risk factor, $71 \%$ had previous injection drug use (IDU) as a risk factor and 14\% had both risk factors.

Three of every 1000 new blood donors in Canada in 1993/1994 had antibodies to HCV (anti-HCV).

Five to $25 \%$ of people with new HCV infections are ill enough to seek medical attention. Estimating the size of the

The Laboratory Centre for Disease Control (LCDC) of Health Canada held a National Meeting on the Prevention and Control of Hepatitis C from December 6-8, 1994. Participants included provincial and territorial epidemiologists and laboratory directors, representatives of professional organizations, individual specialists and representatives of interest groups. This document represents the outcome of that meeting as agreed at the final session; however, certain participants felt that the recommendations should have been more far-reaching in the areas of care, treatment and look-back programs. Copies of the background papers and proceedings of the information sessions held on December 6, 1994 are available from the LCDC (see address in the Correspondence section below). The guidelines and recommendations have been published as a supplement to the Canada Communicable Disease Report (1995; 2152:1-18) and are available over Faxlink and Health Infonet/BBS

Correspondence: The Laboratory Centre for Disease Control, Bureau of Communicable Disease Epidemiology Blood-borne Pathogens Group, Room 1707, Jeanne Mance Building, Tunney's Pasture, Ottawa, Ontario K1A 0K9, telephone 613-954-5205 problem of hepatitis $\mathrm{C}$ through notification is inaccurate and as such the burden of illness and number of deaths from HCV infection in Canada are largely unknown.

\subsection{TRANSMISSION}

The major mode of transmission of HCV in Canada is IDU. Transmission by other routes (eg, sexual) occurs much less frequently but these risks still need to be accurately defined. Since tests became available and use commenced in 1990, it has been shown that HCV can be transmitted by the same routes, although not with the same frequency, as other blood-borne pathogens such as human immunodeficiency virus (HIV) and hepatitis B virus (HBV).

The risk or chance of someone becoming infected with HCV depends on the way in which the person is exposed. An individual exposed through blood or blood product transfusion (before screening of blood donors) is the most likely to become infected, followed by needle sharing in IDU, needlestick injuries (occupational exposure), sexual intercourse and mother to child (vertical transmission).

Blood/blood product transfusion: In Canada, the risk of infection through blood transfusion has been reduced, but not eliminated, by the testing of donors for HCV. After testing for HBV became available in the early 1970s, the virus that was later identified as HCV became the most common cause of post-transfusion hepatitis. Tests for HCV infection have only been available since 1990. The incidence of post-transfusion hepatitis C in the mid-1980s was $3.1 \%$, and fell to $1.3 \%$ by the late 1980s.

Other modes of transmission:

- Rates of infection in those who have ever used injection drugs are at least $30 \%$.

- The risk of sexual transmission is low. The rate is estimated to be about $2.5 \%$ for prolonged sexual exposure (at least 20 years) to infected individuals.

- Estimates of the risk of infection by other routes of trans- 
mission also vary. The occurrence of infection after a needlestick accident has been reported to be approximately $4 \%$.

- The risk of transmission between family members (intrafamilial transmission) and from mother to child (vertical transmission) is probably very low. If the mother is also HIV-positive, the risk of HCV transmission increases.

- HCV has reportedly been found in semen, saliva and breast milk by some researchers but not others. The importance of these findings is not yet clear.

\subsection{CLINICAL ASPECTS}

Five to $25 \%$ of newly infected individuals are symptomatic when first infected; they then recover. As many as $90 \%$ of people newly infected with HCV remain healthy for some time but they continue to carry the virus and may be infectious. These persons are at risk of becoming ill at some time as a result of HCV infection.

The most serious sequelae of HCV infection are cirrhosis and hepatocellular carcinoma (liver cancer). For those who develop sequelae, it has been estimated that it may take 10 years to develop symptoms, 20 years to develop cirrhosis and 30 years to develop cancer of the liver.

Because of the long term nature of $\mathrm{HCV}$ infection, infected individuals may develop and die of diseases unrelated to HCV. This is particularly true of those who acquired HCV as a result of transfusions for life-threatening conditions. Those who acquire $\mathrm{HCV}$ as a result of IDU may also die of other causes before developing complications from HCV infection.

\section{SURVEILLANCE}

\subsection{BACKGROUND}

The purposes of surveillance include the following:

- provision of epidemiologic data, eg, delineation of disease distribution and trends, natural history and burden of illness (morbidity and mortality), identification of risk factors

- identification and prioritization of populations for intervention

- guidance in health care planning

- evaluation of impact of control interventions and prevention programs.

There should be reporting of HCV infection in conjunction with enhanced surveillance to describe further the epidemiology of HCV infection. These twin elements of surveillance are inseparable. In particular, there should be surveillance activities to monitor changes in incidence and patterns of disease.

Nationwide surveillance for HCV infection will be of limited value until there is a way to distinguish acute from chronic infections and until it is possible to estimate $\mathrm{HCV}$-associated morbidity and costs accurately. There is a need to set up pilot projects and sentinel systems to address these concerns and answer other questions on the demo- graphics, risk factors/behaviours and natural history of hepatitis C.

\subsection{RECOMMENDATIONS}

2.2a:

- That HCV infection be added to the list of reportable diseases in all Canadian jurisdictions.

- That reporting be from all laboratories in all Canadian jurisdictions at least.

- That provinces and territories report nationally cases, not tests, and that each HCV-infected individual be reported only once.

- That in addition to laboratories, provinces and territories may include reports from physicians and other sources.

2.2b:

- That the surveillance case definition of HCV infection be a person with laboratory evidence of HCV infection, ie, a positive test for anti-HCV. (This definition does not allow differentiation of acute from chronic infection, of clinical disease from infection, or describe the state of infectivity. This definition recognizes that anti-HCV positivity usually represents nonacute infection but does not exclude acute infection, that anti-HCV positivity usually represents infectiousness and that, in the absence of widely available tests for viral antigens or viral RNA, anti-HCV positivity is the best currently available indicator of the potential to transmit to others.)

\section{2c:}

- That (a minimum of) date of birth, sex and date of report to public health agency be reported nationally in all instances of laboratory evidence of HCV infection.

- That the local Medical Officer of Health ensure that every reported case meets the surveillance case definition.

- That efforts be made at the provincial level to avoid duplicate reporting over time and across health unit jurisdictions.

\section{2d:}

- That a national sentinel system be developed to carry out viral hepatitis surveillance and to gather data on risk factors/behaviours from a population representing the demographics of Canada, including ethnic origin, country of origin and urban/rural mix. (This could be achieved through expansion of the existing Sentinel Health Unit Surveillance System coordinated by LCDC.)

\section{2e:}

- That surveillance be carried out using a variety of other mechanisms to characterize cases (for example, to differentiate acute from nonacute infection and to define the natural history of infection through random sampling of routine case reports for further investigation, sentinel physicians, seroprevalence studies of the general population and persons at high risk).

\section{2f:}

- That a standardized reporting form be developed for enhanced surveillance (ie, surveillance additional to routine 
reporting) to include risk factors/behaviours and elements of natural history in order to facilitate comparisons between jurisdictions.

2.2g:

- That resources commensurate with the tasks outlined above be made available in all jurisdictions.

2.2h Research recommendations: Areas of research to be addressed:

- distinction of acute from chronic disease

- description of natural history of infection

- data links to enumerate/characterize co-infections, eg, HBV and HIV

- standardization of risk factor evaluation across diseases

- easier, more accurate tests for prevalence and screening

- investigation of potential of treatment registry

- the merit of case-finding in selected high risk groups

- HCV-related outcomes of needlestick injury

- health risk behaviours.

\section{BLOOD/BLOOD PRODUCT TRANSFUSION}

\subsection{CURRENT RISK OF HCV TRANSMISSION BY BLOOD/BLOOD PRODUCTS (TRANSFUSION-ASSOCIATED HCV)}

\subsubsection{Background}

Estimates of the current risk of HCV transmission from blood products from window period infections as a result of test characteristics (sensitivity and specificity) using firstgeneration enzyme immunoassay (EIA) tests:

- United States: 1 in 1500 donor exposures (meaning that one person becomes infected as a result of 1500 donations) (1)

- Canada: 1 in 2500 donor exposures (2).

Using second-generation EIA tests:

- United States: 1 in 5000 donor exposures

- Canada: 1 in 7500 donor exposure

with both estimates calculated from first-generation test data but using sensitivity of second-generation tests. These estimates are conservative. Documented risk may be significantly lower. Preliminary results from data being collected in the United States to document risk show no seroconversions to $\mathrm{HCV}$ in 400 patients studied using second-generation tests (1). In Canada, EIA 1.0 and recombinant immunoblot assay (RIBA) 1.0 were used to test blood donations from June 1990, RIBA 2.0 was introduced in May 1991 and EIA 2.0 in May 1992.

Current routinely available tests for $\mathrm{HCV}$ detect anti-HCV. Anti-HCV is a proxy for $\mathrm{HCV}$ infection and potential infectivity.

Although the window period is shortened by thirdgeneration testing, experience in Europe suggests that the introduction of third-generation testing is unlikely to increase significantly the number of HCV-positive donors identified.
The estimated window for HCV infections using secondgeneration testing is 12 to 14 weeks (14-week estimate from European studies, 12-week estimate based on American studies). With third-generation testing, the window is decreased by approximately one week.

The estimated risk of transfusion-associated HCV (TA$\mathrm{HCV}$ ) from a donor in the window period (second-generation testing in place) is 1:62,000 units. This estimate is based on American data using documented seroconversion to $\mathrm{HCV}$ in repeat blood donors and estimates of the window.

There is significant concern regarding the validation of $\mathrm{HCV}$ infection in transfusion recipients identified as $\mathrm{HCV}$ RNA-positive but who remain antibody-negative more than 20 weeks after exposure, ie, false positive laboratory result versus extended window period. Confirmation of infection in this subgroup will be important in defining the limits of the window.

\subsubsection{Recommendations}

3.1.2a:

- That, to lessen the current risk of disease transmission by blood/blood product transfusion, reduction in the use of blood and blood products be given a high priority by agencies in 'the blood system' and agencies responsible for physician practice. Reduction could be achieved by: -development of clinical practice guidelines -physician education -establishment of computerized information systems for look-backs and utilization reviews

-funding and regulation of autologous transfusion programs at Canadian Red Cross centres and hospitals.

\subsection{2b Research recommendation:}

- Accurate determination of the (residual) risk of TA-HCV infection depends on the number of HCV-RNA-positive, anti-HCV-negative donors among current donors. Studies examining this issue should be given priority.

\subsection{IDENTIFICATION OF THOSE AT RISK OF HAVING ACQUIRED TA-HCV}

\subsubsection{Background}

The risk of post-transfusion HCV in the late 1980s was approximately $3 \%$, but it is estimated that a small proportion of Canadians with HCV infection were probably infected through blood/blood product transfusion before 1990 from donors identified since 1990 as being infected with HCV (American estimate less than 5\% [3]).

After consideration of the need to identify those at risk of having acquired HCV infection and after consideration of the different methods by which this might be carried out the following recommendations were made.

\subsubsection{Recommendations}

\subsection{2a:}

- That, because there may be drawbacks as well as benefits in identifying those at risk of infection, before any program is 
undertaken consideration must be given to the potential advantages and disadvantages of a previously transfused individual knowing she/he is HCV-infected.

Benefits include:

- Treatment opportunity. There is some evidence to suggest that patients may respond better to interferon if treated early in the course of their infection. The long term efficacy of interferon treatment on the natural history of disease is unknown and such data will require decades to accumulate.

- The proportion of TA-HCV individuals identified is likely to be higher if done now, rather than in the future, perhaps permitting earlier treatment to a larger number of persons.

- Individuals may make socioeconomic decisions based on knowledge of HCV-seropositivity, eg, job changes/estate planning.

Disadvantages include:

- the stigma associated with the 'HCV-infected' label

- major anxiety related to finding out the disease is present without options for treatment.

3.2.2b:

- That there be a two-pronged general education program for the public and physicians coordinated at the national level and put in place throughout Canada.

- That a public campaign be developed in consultation with all stakeholders including consumer groups.

A general education campaign should include:

- targeting physicians with information on:

-reportability of HCV infection

-identification of infected individuals

-testing procedures

-counselling, treatment and follow-up of infected individuals

-guidelines for use of blood/blood products

(The targets of an education campaign are general practitioners, family physicians, internists, infectious diseases specialists, gastroenterologists, hepatologists, laboratory medicine specialists and other health care workers. Case finding by physicians will lead to notification, which will aid surveillance of HCV infection.)

- providing information to the general public on: $-\mathrm{HCV}$ disease (natural history of infection)

-how the infection disease is transmitted, ie, who is at risk

-risks of past/present IDU and blood/blood product transfusion

-steps to prevent infection (primary prevention)

-steps to prevent spread (secondary prevention)

-self-evaluation of level of personal risk of infection

-advisability of consultation with personal physician if at risk.

\subsubsection{Rationale}

The two-pronged campaign will have the maximum effect of informing those at greatest risk of having acquired infection, ie, blood/blood product transfusion recipients and injec- tion drug users, as well as those who are at greatest risk of acquiring the disease in the future, ie, injection drug users.

The two-pronged education strategy will provide information to all those at risk of HCV infection including those who have had a blood transfusion. This strategy ensures equitable population-wide access to information on the risks and prevention of HCV infection, and the need to seek counselling by those identified at risk.

In contrast, targeted and general look-back programs will only identify previous recipients of blood/blood products, which in the case of a targeted program constitute a small proportion of total HCV infections in Canada. These programs address only secondary prevention and individual case management for blood-related cases. Limiting action to such strategies offers no opportunity for primary prevention of new infections or identification of existing $\mathrm{HCV}$ infections (nonblood-related) in the general population.

The Canadian Red Cross Society has recommended targeted and general look-back programs in an attempt to identify recipients of blood transfusion or blood products from donors identified as being RIBA 2.0-positive since 1990, and to inform them that they may have been infected with HCV and that they should be tested. Persons thus identified have a high likelihood of being HCV-infected and therefore may benefit from appropriate case management. With regard to disease control in the Canadian population, however, there is marginal utility of transfusion look-back programs in identifying those at risk of HCV infection compared with a general education program.

NOTE: a) Any look-back program must be associated with a careful implementation plan including ensuring that adequate resources are available for agencies involved such as the Canadian Red Cross, hospital blood banks and laboratories providing anti-HCV testing.

b) Cost/benefit analyses should be put in place to evaluate the effectiveness of any look-back programs implemented, with rapid reallocation of resources if effectiveness is poor. A better estimate of the number of surviving TA-HCV infected individuals in Canada is required for these analyses.

\subsubsection{Other issues}

3.2.4a: The working group that considered issues of bloodborne transmission during the meeting discussed further programs that might be carried out in addition to a general education program. The group felt that the following prioritization might be considered.

1) Targeted look-back (standard, Canadian Red Crossinitiated identification of infected donors) - recipients of previously donated blood from donors found to be anti-HCV positive would be targeted for anti-HCV testing.

2) Encouraging the routine, practice-based review of patient transfusion histories by physicians and discussion regarding anti-HCV testing with all patients who received blood before the introduction of EIA 2.0 testing in May 1992. 
3) Trace-back from cases of transfusion-associated HCV infection with notification of Canadian Red Cross and thence identification of donors leading to a targeted look-back.

4) Targeted look-back (extended), ie, persons identified by any agency as being anti-HCV positive are questioned about prior blood donation and, where necessary, targeted look-back is undertaken.

5) Testing of stored specimens taken from donors before routine second-generation testing began in 1992 (Canadian Red Cross-initiated, with subsequent targeted look-back if appropriate).

\subsection{4b Recommendation:}

- That routine testing of transfusion recipients for anti-HCV at six months or longer post-transfusion not be carried out. (The current risk of TA-HCV infection is estimated to be low given present blood screening procedures. Further data to document this risk in the setting of second-generation anti-HCV testing will be available from the United States before similar studies are initiated in Canada. Therefore, the routine testing of all prospective transfusion recipients for anti-HCV before and after transfusion is a high cost, low yield endeavour.)

\subsection{4c Research recommendation:}

- That high priority be given to initiating pilot studies to look at the necessity, cost, feasibility and yield of hospitalinitiated identification of transfusion recipients in multiply transfused populations with long life expectancy after transfusion, eg, transfused neonates and pediatric surgery patients.

\section{MODES OF TRANSMISSION OTHER THAN BLOOD/BLOOD PRODUCT TRANSFUSION}

\subsection{HEALTH CARE SETTINGS}

\subsubsection{Recommendations}

- That recommendations for the prevention of blood-borne infections in health care settings that have already been made $(4,5)$ be applied to HCV.

- That universal precautions be regarded as the minimum standard of practice for preventing transmission of bloodborne pathogens in all health care settings. Universal precautions must be clearly and consistently defined wherever they are described.

\subsection{1a HCV testing for health care workers:}

- That mandatory testing of health care workers is not justified based on current scientific evidence.

- That health care workers who have had a previous significant exposure (exposure to blood and body fluids via nonintact skin or needlestick injury) or who have personal risk factors, eg, IDU, be encouraged to seek HCV testing voluntarily.

- That voluntary testing of health care workers who have no personal risk factors, based on perceived or potential occupational risk of transmitting HCV (eg, persons performing invasive procedures), not be done.

\subsection{1b HCV-infected health care workers:}

- That any health care worker with an infectious disease that could put a patient at risk be encouraged to seek medical evaluation voluntarily with respect to the potential for transmission of the infection to patients. Seeking medical evaluation is a fundamental ethical principle for health care workers infected with HCV.

- That current guidelines and legislation dealing with confidentiality be reinforced and followed.

- That reporting of anti-HCV positive health care workers to their professional organizations be done only within the requirements of current legislation.

- That medical evaluation of an infected health care worker be the responsibility of the health care worker's primary care physician. Primary care physicians who care for HCV-infected health care workers are encouraged to seek advice on assessment of the risk of infection transmission in the health care setting.

- That a consultation mechanism that can be easily accessed by a primary care physician be established, ideally in each province. (This mechanism should ensure confidentiality and allow for input from public health, licensing bodies and/or professional associations, experts in infectious diseases and infection control, and others as judged appropriate to the situation. Participants in this process need not know the health care worker's identity. An existing provincial reporting/consultation system may be adapted to serve as the consultation mechanism.)

- That criteria used to assess seropositive health care workers include the medical evaluation, knowledge, application of infection control practices and risk of injuries from sharp objects in the context of the individual's occupation.

- That supportive nonthreatening programs through licensing and/or professional organizations be developed to assist seropositive health care workers whose practices are modified because of their infection status. Career counselling and, if necessary, job retraining should be encouraged to promote the use of the health care worker's skills and knowledge.

\subsection{1c Disclosure of infected health care worker's status:}

- That routine disclosure of an infected health care worker's serological status is not justified.

- That a patient be notified when a significant exposure to an infected health care worker has occurred. There is no need to disclose the identity of the exposure source.

\subsection{1d Occupational postexposure management:}

- That because a significant exposure to blood or body fluids from an infected person may lead to HCV infection, all such exposures be fully documented. 
- That a working group be established to develop a national postexposure protocol for $\mathrm{HCV}$.

- That immune serum globulin not be used in postexposure management.

- That HCV be added to the national surveillance program for occupational postexposure to HIV.

4.1.1e Institutional issues:

- That, because there are no perceived benefits of routine screening for $\mathrm{HCV}$, it not be carried out in an institutional setting, eg, acute and long term care and correctional facilities.

\subsection{PERSONAL SERVICES}

\subsubsection{Recommendation}

- That, because HCV and other blood-borne infections are transmissible through personal services such as tattooing, body piercing and electrolysis, national guidelines and a national strategy be developed for the prevention of blood-borne infections in these settings.

\subsection{IDU}

\subsubsection{Recommendations}

- That, because IDU is the major mode of transmission of $\mathrm{HCV}$, all injection drug users be counselled according to current guidelines regarding prevention of HIV transmission, regardless of serostatus.

- That education regarding the use of clean needles be expanded to include all equipment and paraphernalia.

- That access to specific programs such as needle exchange and detoxification be improved if the future burden of this disease is to be reduced.

\subsection{ACCIDENTAL NEEDLESTICKS}

\subsubsection{Recommendation}

- That the proposed working group on national postexposure protocol for $\mathrm{HCV}$ consider community needlestick accidents. (A consensus was not reached on testing for $\mathrm{HCV}$ in community-acquired needlestick injuries. Each circumstance will have to be considered separately before national guidelines are produced).

\subsection{SEXUAL TRANSMISSION}

\subsubsection{Recommendations}

4.5.1a:

- That whereas the magnitude of the risk of sexual transmission of HCV has not been established, but appears to be much lower than that of HIV or HBV, current knowledge does not warrant partner notification/contact tracing; however, HCV-infected persons have a personal responsibility to inform potential sexual partner(s) that there is a risk of infection (up to $2.5 \%$ with long term [more than 20 years'] sexual exposure). 4.5.1b HCV-positive individuals with multiple sexual partners:

- That, despite the lack of good supporting scientific data on the efficacy of risk reduction activities in the prevention of transmission of $\mathrm{HCV}$, an $\mathrm{HCV}$-infected person be provided with information that may reduce the risk of sexual transmission. This information should include the range of safer sex practices.

4.5.1c HCV-positive individuals in long term sexual relationships:

- That, because the risk of transmission may increase with repeated sexual exposures, long term sexual partners be offered testing by their physician.

- That, despite the lack of good supporting scientific data on the efficacy of risk reduction activities in the prevention of transmission of $\mathrm{HCV}$, an $\mathrm{HCV}$-infected person be provided with information that may reduce the risk of sexual transmission. This information should include the range of safer sex practices. Information should be provided on the estimates of risk.

\subsection{MOTHER TO INFANT TRANSMISSION}

\subsubsection{Background}

Because current data indicate that vertical, intrapartum and horizontal transmission are rare, and because the mechanism (in utero, parturition or breast feeding) has not been established and there is a lack of information, specific recommendations against pregnancy and breast feeding cannot be made.

\subsubsection{Recommendations}

- That information on the risks as they are presently known be given to HCV-infected women of childbearing age.

- That immune serum globulin not be given to infants of $\mathrm{HCV}$-infected mothers postpartum.

\subsection{HOUSEHOLD TRANSMISSION}

\subsubsection{Background}

There are insufficient data to make specific recommendations regarding $\mathrm{HCV}$ transmission to household contacts.

\subsubsection{Recommendation}

- That because of a theoretical risk, advice based on standard guidelines for avoiding exposure to blood be provided, eg, avoidance of the sharing of razors, toothbrushes and other personal hygiene items.

\subsection{IDENTIFICATION OF PERSONS AT RISK OF HAVING ACQUIRED HCV BY MODES OTHER THAN BLOOD/BLOOD PRODUCT TRANSFUSION}

\subsubsection{Recommendations}

- That professional societies make a concerted effort to educate health care providers about: 
-known and potential risks for HCV infection

-the need to ascertain complete behaviour risk histories from patients

-the appropriate evaluation of at-risk patients for evidence of infection

-current status of investigations on use of alpha-interferon to treat $\mathrm{HCV}$.

\subsection{RESEARCH RECOMMENDATIONS}

Further research is required on the risk of:

- sexual transmission

- household transmission

- maternal to child transmission through:

-breast feeding

-parturition

-caesarian section

-delivery

-amniocentesis

- survival of virus in the environment (including effective disinfectants); however, it is recognized that this is currently not technically possible

- viral infectivity (serological assays), ie, quantification of infectious dose.

\section{CASE MANAGEMENT}

\subsection{PERSONS WITH INCREASED LIKELIHOOD OF HCV INFECTION, INDICATORS OF RISK}

\subsubsection{Guidelines}

Risk indicators include:

- history of previous IDU

- known blood/blood products transfusion in Canada before May 1992 (the date after which both second-generation EIA and immunoblot [RIBA 2.0] tests of blood donors were in use)

- multiple transfusions (at any time), eg, those with thalassemia and hemophilia

- needlestick injury associated with a known HCV-infected source

- children of HCV-infected mothers

- organ and tissue transplant recipients

- abnormal aminotransferases

- cryptogenic cirrhosis or alcoholic cirrhosis

- hepatocellular carcinoma (hepatoma)

- mixed cryoglobulinemia

- glomerulonephritis of unknown origin

- porphyria cutanea tarda.

Note: Clinical judgement is needed when making a decision to test individuals who are infected with other blood-borne pathogens, eg, HBV acquired through vertical transmission.

\subsection{FURTHER INVESTIGATION OF HCV-INFECTED PERSONS}

\subsubsection{Background}

Individuals will come to medical attention through two routes: by identification of elevated aminotransferases at insurance or routine examination; or by identification of anti-HCV positivity through screening or investigation of illness. The follow-up for each group is different.

\subsubsection{Guidelines}

- If identified because aminotransferases are elevated: $-\mathrm{HCV}$ testing as part of diagnostic work-up.

- If identified anti-HCV positive by screening or disease investigation:

-aspartate aminotransferase, alanine aminotransferase (ALT), alkaline phosphatase, gamma glutamyltransferase

-liver function tests, ie, albumin, international normalized ratio (INR) of prothrombin time, bilirubin.

- Before testing an individual, there must be informed consent including communication of the nature and consequence of an HCV test (including provisions for pre- and post-test counselling).

- Professional bodies should prepare guidelines regarding pre- and post-test counselling and these guidelines should be made available to physicians who order HCV tests (as for HIV).

- Physicians must inform patients of their anti-HCV antibody status as soon as practically possible once the information becomes available.

Note: Liver biopsy is strongly suggested if ALT remains elevated for more than six months or if there is clinical evidence of cirrhosis. The biopsy findings should be reported in terms of severity of disease according to the scheme of Desmet et al (6), not using the outdated nomenclature of 'chronic active' and 'chronic persistent' hepatitis.

\subsection{PROGNOSIS}

\subsubsection{Guidelines}

- The majority of HCV-infected individuals remain persistently infected (ie, HCV RNA-positive).

- Per decade, it is estimated that $10 \%$ of HCV-infected individuals develop cirrhosis.

- Twenty per cent of HCV-infected individuals who are evaluated at one point have biopsy-verified cirrhosis (cross-sectional point prevalence studies).

- The proportion of HCV-infected individuals who develop hepatic decompensation each year is unknown.

- The risk of cirrhosis increases with excessive alcohol use (threshold not known); thus, alcohol consumption should be reduced to a maximum of not more than one alcoholic beverage per day.

- The risk of cirrhosis increases with:

-duration of infection

-severity of disease on biopsy 
-concurrent infection with HBV, delta-hepatitis, HIV or hepatitis A virus (HAV).

\subsection{HEPATOCELLULAR CARCINOMA}

\subsubsection{Guidelines}

- The risk of hepatocellular carcinoma increases with all types of cirrhosis (substantial increases in hepatocellular carcinoma incidence are projected for the next 10 to 20 years associated with both HBV and HCV).

- In North America hepatocellular carcinoma is frequently associated with cirrhosis due to HCV. Hepatocellular carcinoma is rare in hepatitis $\mathrm{C}$ without cirrhosis and it is uncertain whether hepatitis $\mathrm{C}$, without cirrhosis, confers additional risk of this cancer.

\subsection{OTHER DISEASES}

\subsubsection{Guideline}

Other diseases (eg, mixed cryoglobulinemia, glomerulonephritis and porphyria cutanea tarda) associated with HCV infection occur only in a small subpopulation. It is not necessary to investigate routinely for these diseases.

\subsection{FOLLOW-UP OF IDENTIFIED HCV-INFECTED INDIVIDUALS}

\subsubsection{Guidelines}

- Follow-up with yearly ALTs if the liver aminotransferases are persistently normal, ie, on three to four serial tests within one year.

- Follow-up with six monthly tests of bilirubin, albumin, INR of prothrombin time and ALTs if aminotransferases are elevated and treatment is not currently indicated.

- If an individual has established cirrhosis, the risk of liver failure requires specialist follow-up because of complications such as ascites, variceal bleeding and hepatic encephalopathy.

- All individuals infected with HCV who are not immune to HBV should be offered HBV immunization to prevent any further liver damage. For the same reason, those who are at high risk of HAV should be offered HAV immunization.

- Other diseases associated with HCV infection should be investigated when clinically relevant.

- Routine case finding for hepatocellular carcinoma needs further study and cannot be routinely recommended with the current state of knowledge.

- Nucleic acid tests, eg, polymerase chain reaction (PCR), are not part of routine follow-up, but when tests are standardized and validated they may become a useful part of management, especially in individuals with normal aminotransferases.

\subsubsection{Recommendation}

- Because there is a need to anticipate both the technological and human resource needs that will evolve as increasing numbers of Canadians are diagnosed with HCV infection, a mechanism should be found to address issues related to concerns of long waiting lists and a potential lack of related specialists.
Specific issues to be addressed include, but should not necessarily be limited to, the number of practising specialists with expertise in hepatitis (primarily hepatologists, gastroenterologists, infectious disease physicians and medical microbiologists), the number of fellowship positions with appropriate hepatology training, the amount of hepatology training in gastroenterology training programs, training of and information sharing with primary care health care workers (family physicians and nursing) and the technological and physical plant support needed to manage $\mathrm{HCV}$-infected persons adequately.

\subsection{TREATMENT}

\subsubsection{Guidelines}

- The published consensus statement $(7,8)$ on the treatment of chronic hepatitis in individuals infected with HCV remains a good guideline given the current state of knowledge.

- Nonetheless, interferon treatment is not indicated for most subjects and additional studies, both underway and planned, may soon result in changes to these recommendations.

- HCV is the second-most common reason for liver transplantation. Patients with HCV and liver failure remain candidates for liver transplantation. Transplantation is usually successful and recurrences of $\mathrm{HCV}$ infection in the transplanted liver usually result in mild disease.

\subsubsection{Recommendations}

- That when treatment is indicated there is to be equity of access to interferon therapy in all provinces across Canada.

- Because liver transplantation is currently limited by donor availability and the need for liver transplantation may outstrip the capacity to provide donor livers in the next 10 to 20 years, there should be discussions among governments, professionals with an interest in liver disease (eg, through the Canadian Association for the Study of the Liver or the Canadian Liver Foundation) and funding agencies to set an agenda for research in $\mathrm{HCV}$, including the need to support, plan and fund adequate studies to clarify optimum therapy, indicators of therapeutic efficacy (ie, monitoring therapy) and indicators to predict how individuals will respond to therapy (eg, HCV RNA levels and genotype).

\subsection{RESEARCH ISSUES}

Issues to be addressed by research include the following:

- technology for diagnosis, immediate management and follow-up of HCV-infected individuals

- quality of life issues throughout the natural history of hepatitis C

- determination of the efficacy and cost-efficiency of screening for hepatocellular carcinoma

- treatment:

-definition of optimal therapy

-predictors of response including overall morbidity and mortality

-evaluation of the efficacy of delivery of services, and the effect of various interventions 
-natural history of infection

-prognosis of ALT normal subjects

-long term natural history for those treated and not treated with interferon

-prognosis of PCR-negative, anti-HCV-positive subjects

-prognosis for transfusion-related HCV in recently infected persons

-epidemiology of hepatitis $\mathrm{C}$ in aboriginal populations -epidemiology of hepatitis $\mathrm{C}$ in high risk groups with an emphasis on injection drug users since they will, in time, constitute the vast majority of infected individuals -natural history in those infected at a young age and utility of early identification and treatment.

\subsection{RESEARCH RECOMMENDATION}

- It is strongly suggested that a national database be established to provide the framework for studies of the natural history of this disease.

\section{LABORATORY DIAGNOSIS}

\subsection{TESTING}

\subsubsection{Recommendations}

- That EIAs remain the test of choice for initial assessment of specimens. Currently available EIA tests are based on the immunological response to infection and are an accurate, indirect measure of infection and infectivity. They are the most practical tests for the first-line detection of HCV infections and are more than $90 \%$ sensitive for the detection of established infection.

- That, because of false positive reactions, supplemental testing be used. False positive EIA test results do occur. In low risk populations (ie, blood donors, patients tested because of previous transfusion history), the proportion of reactive tests that are falsely positive can be greater than $50 \%$. The specific nature of the supplemental test(s) used varies according to circumstances.

- Tests based on nucleic acid detection (eg, PCR) can be useful in specific circumstances. The EIA test has certain limitations. In immunocompromised persons, including dialysis patients, HIV-infected persons and transplant recipients, the antibody response to infection may be blunted, and in acute infections there is a window between infection and appearance of detectable antibodies of weeks to months. The window (HCV RNA-positive, anti-HCV-negative) in immunocompetent individuals is five to eight weeks, on average. Nucleic acid detection tests can detect acute infection within one to two weeks of exposure. In children born to infected mothers, antibody tests do not permit diagnosis of infection in the first months of life because of passive transfer of maternal antibodies.

- Because nucleic acid testing is technically complex, the technique needs further standardization and requires critical specimen handling and processing for accurate determination of the results.

- That, although nucleic acid-based testing of HCV can shorten the time to diagnosis of acute HCV infection (ie, reduce the window), nucleic acid tests not be used routinely because the results do not affect the management of patients or the public health response; however, in immunocompromised persons and children of infected mothers nucleic acid tests may be the only way of detecting HCV infection.

\subsection{QUALITY ASSURANCE}

\subsubsection{Recommendations}

- That all laboratories providing serological testing services for HCV infection have access to and participate in external quality control programs for EIA and immunoblot assays.

- That an external quality control program for nucleic acid detection technologies be developed. All laboratories providing such services should participate in this program.

\subsection{RECOMMENDED ALGORITHMS}

\subsubsection{Screening*}

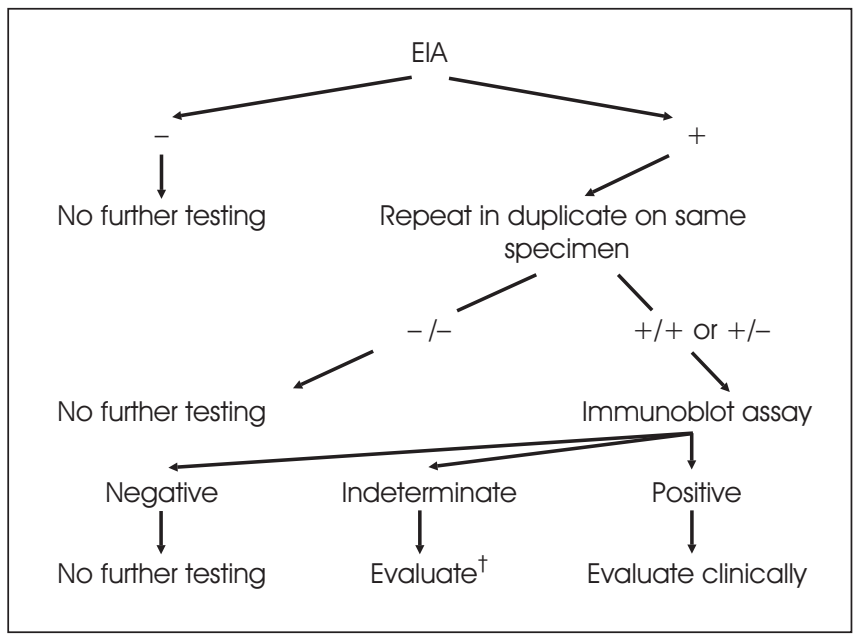

*Applicable also to blood, organ, tissue and sperm donors; ${ }^{\dagger}$ Nucleic acid detection methods can be useful in resolving indeterminate immunoblot results. EIA Enzyme immunoassay

\subsubsection{Acute infections}

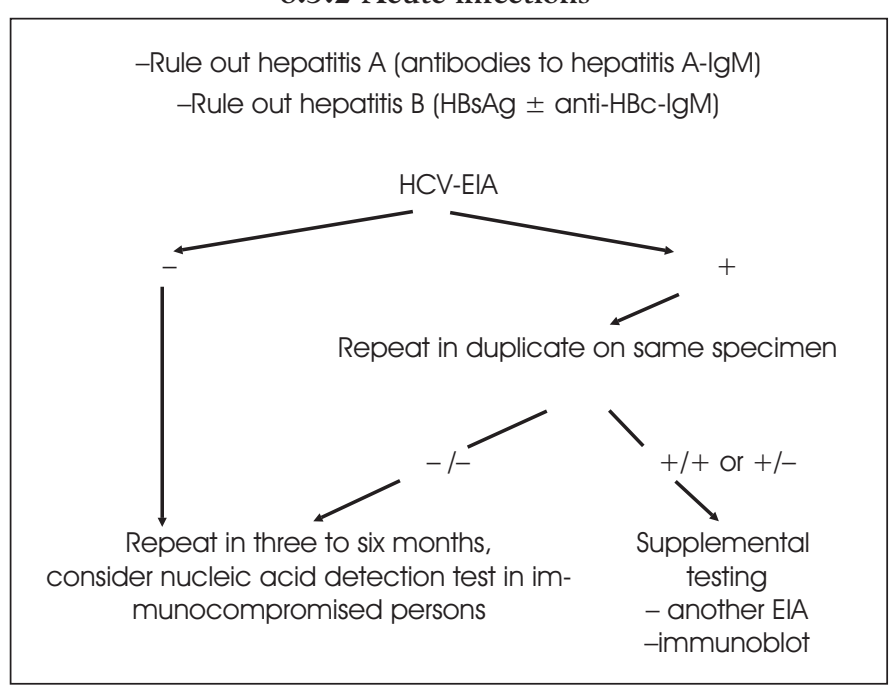

anti-HBc Antibodies to hepatitis B core antigen; EIA Enzyme immunoassay; Ig Immunoglobulin; HBsAg Hepatitis B surface antigen; HCV Hepatitis $\mathrm{C}$ virus 


\section{RESEARCH RECOMMENDATIONS}

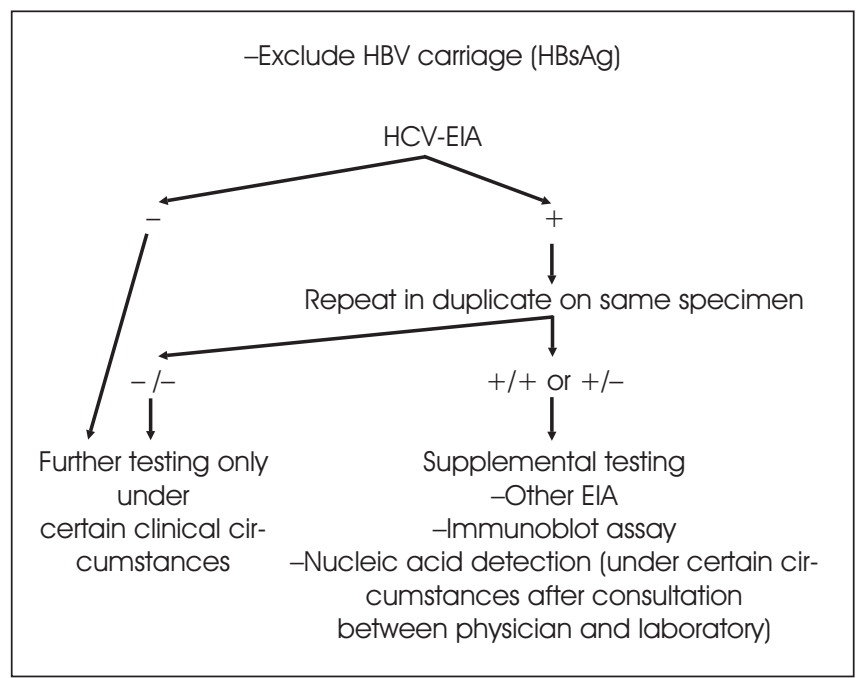

EIA Enzyme immunoassay; HBsAg Hepatitis B surface antigen; HBV Hepatitis B virus; HCV Hepatitis C virus

\section{REFERENCES}

1. Busch M. Transmission of blood and blood products. In: Proceedings of a National Meeting on the Prevention and Control of Hepatitis C, Ottawa, December 6-8, 1994. (In press)

2. Blajchman MA, Bull SB, Feinman SV. Post-transfusion hepatitis: impact of non-A, non-B hepatitis surrogate tests. Lancet 1995;345:21-5.

3. Alter M. Current epidemiology. In: Proceedings of a National Meeting on the Prevention and Control of Hepatitis C, Ottawa, December 6-8, 1994. (In press)

4. Laboratory Centre for Disease Control. Bloodborne pathogens in the health care setting: Risk for transmission. Can Commun Dis Rep 1992:18:177-24.

\section{Questions:}

- Does acute infection without antibody response exist in immunocompetent persons? If so, how frequent is it?

- What are the roles of viral load determination and genotype determinations in the clinical care of patients?

- What is the genotype distribution in infected Canadians?

- What is the utility of pooling sera for the purpose of population screening studies using antibody techniques?

- Can double EIA algorithms be used to determine infection status of screened individuals?

- What is the optimal follow-up protocol for infants of infected mothers?

- Are saliva and urine useful as specimens for seroepidemiological studies?

- Is HCV nucleic acid quantification and detection of specific viral HCV genotypes useful in establishing prognosis and assessing the efficacy of HCV treatment programs?

5. Laboratory Centre for Disease Control. Preventing the transmission of blood-borne pathogen infections in public service facilities. (In press)

6. Desmet VJ, Gerber M, Hoofnagle JM, Manns M, Scheuer PJ. Classification of chronic hepatitis: diagnosis, grading and staging. Hepatology 1994;19:1513-20.

7. The CASL Hepatitis Consensus Group. Treatment of chronic viral hepatitis with alpha-interferon: A consensus conference report. Can J Infect Dis 1994;5:107-12.

8. The CASL Hepatitis Consensus Group. Treatment of chronic viral hepatitis with alpha-interferon: A consensus conference report. Can J Gastroenterol 1994;8:179-84. 


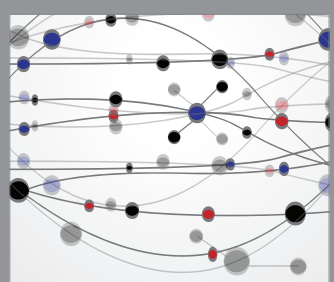

The Scientific World Journal
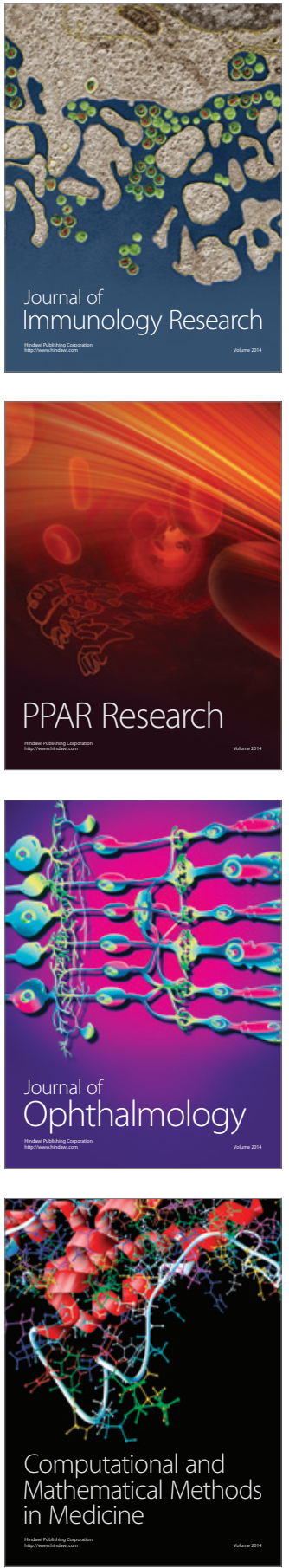

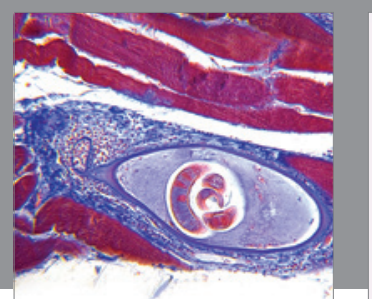

Gastroenterology Research and Practice

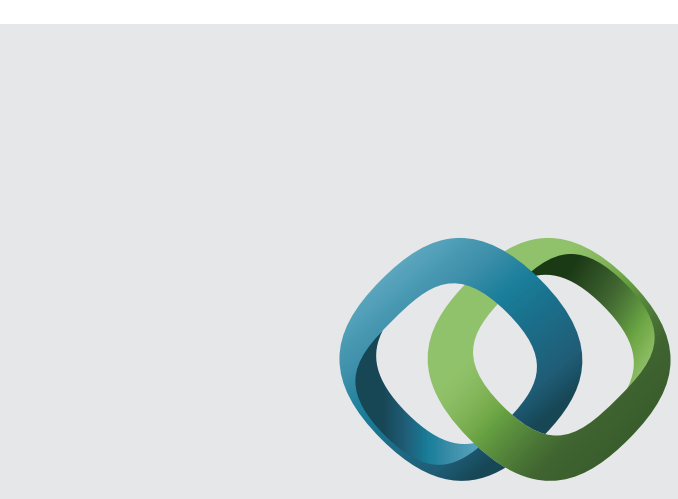

\section{Hindawi}

Submit your manuscripts at

http://www.hindawi.com
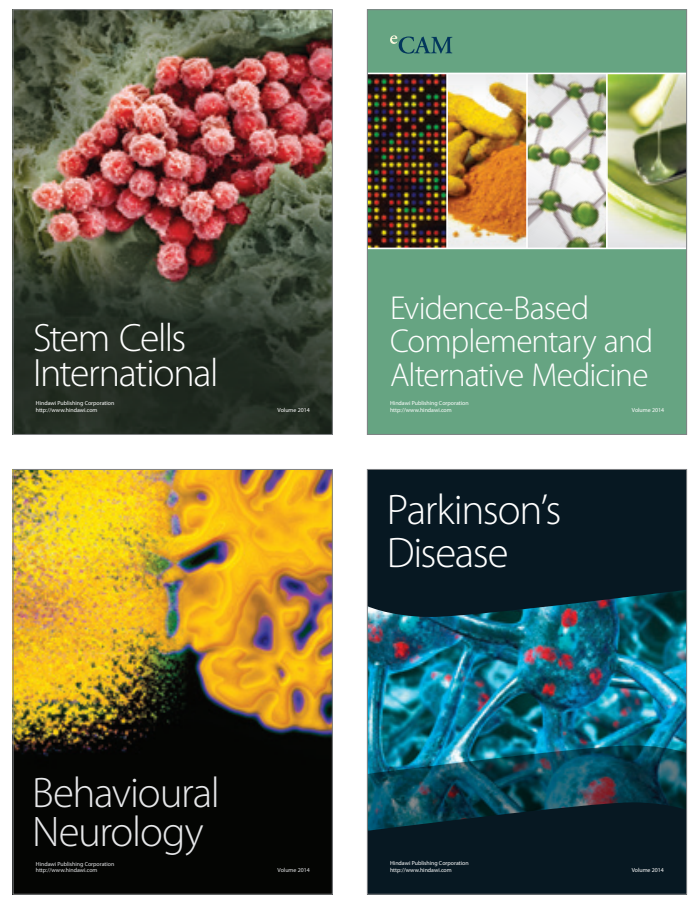
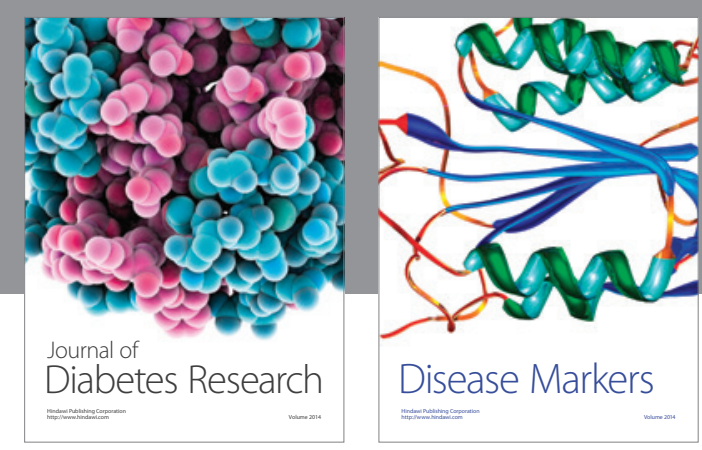

Disease Markers
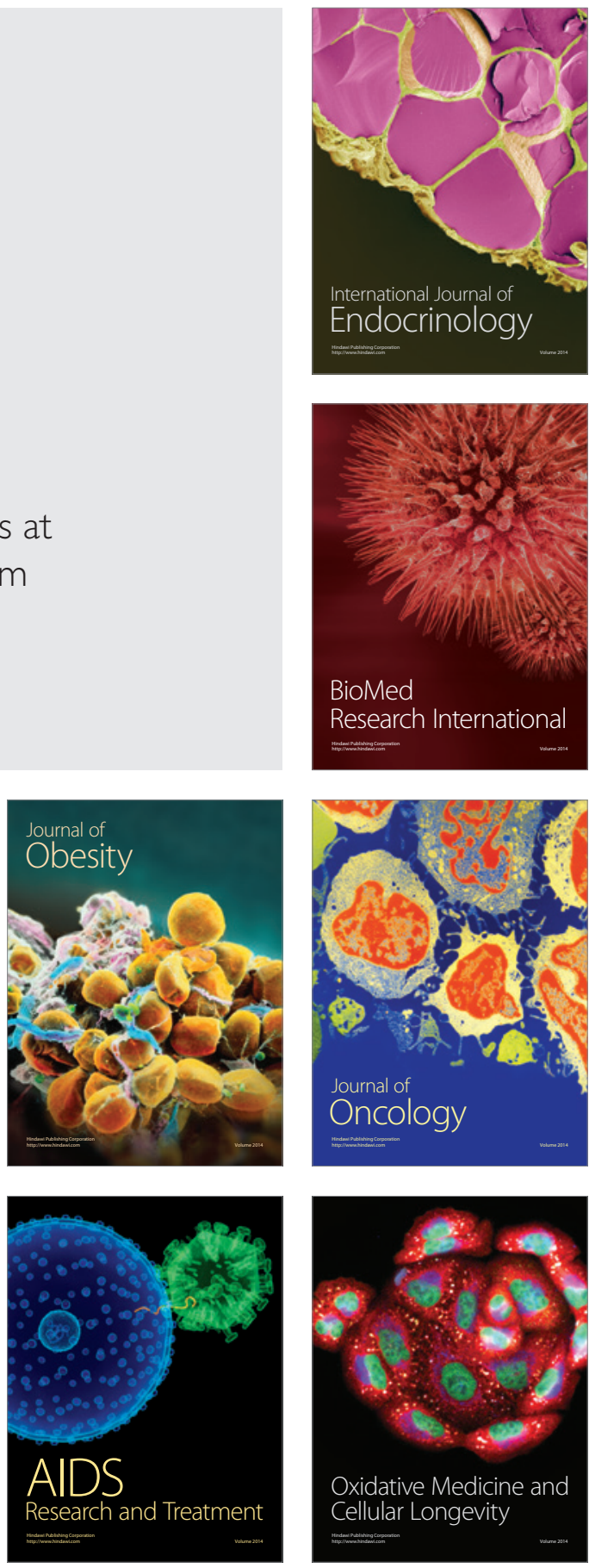